\title{
Two-species flocking particles immersed in a fluid
}

\author{
Young-Pil Choi And Bongsuk Kwon
}

\begin{abstract}
We present a new particle-fluid model describing the motion of two-species flocking particles immersed in an incompressible viscous fluid. The flocking particles are directly affected by the incompressible fluid through a drag force, and they are also coupled with each other via the fluid. On the other hand, the two-species particles are coupled with each other via the viscous fluid. For this proposed model, we show the global existence of a unique strong solution when the initial data is sufficiently small, and we also investigate the large-time behavior of the solutions under suitable conditions.
\end{abstract}

\section{Introduction}

In this paper, we present a new model for the two-species flocking particles interacting with an incompressible fluid. The model consists of the Vlasovtype equations with flocking force terms for the particles and the incompressible Navier-Stokes equations for the fluid. The flocking particles are directly affected by the Navier-Stokes equations through a drag force, and they are also coupled with each other via the fluid. For the proposed model, we first show the existence, uniqueness and regularity of the strong solutions when the initial data is sufficiently small, and investigate the large-time behavior of the solutions using a priori estimates.

More specifically, let $f_{i}=f_{i}(x, \xi, t), i=1,2$ be the one-particle distribution function of the flocking particles at the phase-space position $(x, \xi) \in$ $\Omega \times \mathbb{R}^{3}$ at time $t$, and $u=u(x, t)$ be the bulk velocity of the incompressible fluid. Here $\Omega$ is either a periodic space $\mathbb{T}^{3}:=\mathbb{R}^{3} / \mathbb{Z}^{3}$ or a whole space $\mathbb{R}^{3}$. Then the particles and fluid are governed by the following equations:

$$
\begin{aligned}
& \partial_{t} f_{1}+\xi \cdot \nabla_{x} f_{1}+\nabla_{\xi} \cdot\left(\left(F_{a}^{1}\left[f_{1}\right]+F_{d}[u]\right) f_{1}\right)=0, \quad(x, \xi) \in \Omega \times \mathbb{R}^{3}, \quad t>0, \\
& \partial_{t} f_{2}+\xi \cdot \nabla_{x} f_{2}+\nabla_{\xi} \cdot\left(\left(F_{a}^{2}\left[f_{2}\right]+F_{d}[u]\right) f_{2}\right)=0, \quad(x, \xi) \in \Omega \times \mathbb{R}^{3}, \quad t>0, \\
& \partial_{t} u+u \cdot \nabla_{x} u+\nabla_{x} p-\mu \Delta_{x} u \\
& \quad=-\int_{\mathbb{R}^{3}} F_{d}[u]\left(f_{1}+f_{2}\right) d \xi, \quad \nabla_{x} \cdot u=0, \quad x \in \Omega, t>0,
\end{aligned}
$$


subject to initial data:

$$
\left.\left(f_{1}, f_{2}, u\right)\right|_{t=0}=\left(f_{10}, f_{20}, u_{0}\right), \quad \nabla_{x} \cdot u_{0}=0 \quad \text { in } \Omega \times \mathbb{R}^{3},
$$

and, in the case of $\Omega=\mathbb{R}^{3}$, the end state condition for $u$ is imposed:

$$
u(x) \rightarrow u_{\infty} \quad \text { as } \quad|x| \rightarrow \infty .
$$

Here $F_{a}^{i}, i=1,2$, and $F_{d}$ are the flocking alignment forces and the drag force per unit mass, respectively:

$$
\begin{aligned}
F_{a}^{i}\left[f_{i}\right](x, \xi, t) & :=\int_{\Omega \times \mathbb{R}^{3}} \psi_{i}(x, y)\left(\xi_{*}-\xi\right) f_{i}\left(y, \xi_{*}, t\right) d \xi_{*} d y, \quad i=1,2, \\
F_{d}[u](x, \xi, t) & :=u(x, t)-\xi
\end{aligned}
$$

where the communication weight function $\psi_{i}: \Omega \times \Omega \rightarrow \mathbb{R}_{+}$is a $\mathcal{C}^{1}$-function satisfying the symmetric and nonnegative conditions:

$$
\psi_{i}(x, y)=\psi_{i}(y, x), \quad \psi_{i} \geq 0, \quad i=1,2 .
$$

For the communication weight functions, there are various possibilities to adopt. For example, in the case of $\Omega=\mathbb{R}^{3}$, a regular kernel as in the CuckerSmale models can be chosen:

$$
\psi_{i}(x, y)=\frac{\alpha_{i}}{\left(1+|x-y|^{2}\right)^{\beta_{i} / 2}}, \quad \alpha_{i}>0, \quad \beta_{i} \geq 0, \quad i=1,2 .
$$

Throughout the paper, we assume $\mu=1$ and $u_{\infty}=0$. In fact, a more general condition on the viscosity coefficient $\mu>0$ does not yield any difficulties for our analysis, and the assumption on the far-field $u_{\infty}$ is reasonable due to the Galilean invariance for the fluid equations.

The collective behavior of the interaction particle systems such as flocking, aggregation, and synchronization has received a bulk of attention from various research fields arising in physics, biology, robotics, control theory and other disciplines $[1,5,10,14,15,16,24,27,28,30]$. The interaction between the flocking particles and fluids is first studied in [2]. They considered the kinetic equation for the Cucker-Smale flocking particles coupled with the incompressible Navier-Stokes equations, and showed the global existence of weak solutions. We refer readers to [2] and references therein for a detailed description of the modeling and the related literature. Later in [3] they showed the global strong solutions for sufficiently small and regular initial data, and the large-time behavior of the classical solutions in 
three space dimensions was obtained under suitable assumptions. Concerning two dimensions case, Choi and Lee [11] established the global existence of weak and strong solutions. Unlike the three dimensions case, the smallness assumption on the initial data has been removed to show the global existence of strong solutions. More recently, the interaction between the Cucker-Smale type flocking particle and the compressible viscous fluid is studied in [4]. For the other related particle interacting with fluid, we refer to $[6,7,8,9,13,17,22,26]$.

We now extend the previous result for the one-species problems to the case for two particle species. We would like to describe the situation in which more than one type of flocking particle interacting with each other in the fluid. Two-species models have many applications such as pedestrian flows [29], opinion formation between two groups with different leanings [18, 19], and so on. A mathematical study of existence, stability, finite-time blow up, and the large-time behavior for two competitive populations of biological species which are attracted by random diffusion and chemotaxis is another recent active research area $[12,20,23,31]$. We also refer to [21, 25] for nonlocal interaction PDEs with two-species.

Our first result is concerned with the global existence of a unique strong solution to the system (1.1)-(1.4) when the initial data is sufficiently small and regular. For this purpose, we do not restrict the communication weight function to the specific ones. Rather various alignment forces can be adopted for various physical situations. In particular, as we mentioned before, the regular alignment force as in the Cucker-Smale model can be considered.

Theorem 1.1. Suppose that the initial data $f_{10}$ and $f_{20}$ have a compact support in position and velocity. For $T \in(0, \infty)$, there exists a positive constant $\epsilon_{0}$ such that if $\left\|f_{10}\right\|_{W^{1, \infty}\left(\Omega \times \mathbb{R}^{3}\right)}+\left\|f_{20}\right\|_{W^{1, \infty}\left(\Omega \times \mathbb{R}^{3}\right)}+\left\|u_{0}\right\|_{H^{2}(\Omega)}<\epsilon_{0}$, the system (1.1)-(1.4) has a unique strong solution $\left(f_{1}, f_{2}, u\right)$ satisfying

$$
\begin{aligned}
& \text { (i) } f_{1}, f_{2} \in W^{1, \infty}\left(\Omega \times \mathbb{R}^{3} \times(0, T)\right) \\
& \text { (ii) } u \in \mathcal{C}\left([0, T] ; H^{2}(\Omega)\right) \cap L^{2}\left(0, T ; H^{3}(\Omega)\right) \quad \text { and } \\
& u_{t} \in \mathcal{C}\left([0, T] ; L^{2}(\Omega)\right) \cap L^{2}\left(0, T ; H^{1}(\Omega)\right), \\
& \text { (iii) } p \in \mathcal{C}\left([0, T] ; H^{1}(\Omega)\right) \cap L^{2}\left(0, T ; H^{2}(\Omega)\right) .
\end{aligned}
$$

Our second result concerns the large-time behavior of the strong solutions to the system (1.1)-(1.4) for the periodic spatial domain, i.e., $\Omega=\mathbb{T}^{3}$. To this end, we first introduce a total energy-variance function $\mathcal{E}(t)$ :

$$
\mathcal{E}(t):=\frac{1}{2}\left(\int_{\mathbb{T}^{3} \times \mathbb{R}^{3}}\left|\xi-\xi_{c}^{1}\right|^{2} f_{1}+\left|\xi-\xi_{c}^{2}\right|^{2} f_{2} d x d \xi\right.
$$




$$
\left.+\int_{\mathbb{T}^{3}}\left|u-u_{c}\right|^{2} d x+\frac{1}{2}\left|u_{c}-\left(\xi_{c}^{1}+\xi_{c}^{2}\right)\right|^{2}\right),
$$

where

$$
\xi_{c}^{i}(t):=\frac{\int_{\mathbb{T}^{3} \times \mathbb{R}^{3}} \xi f_{i} d x d \xi}{\int_{\mathbb{T}^{3} \times \mathbb{R}^{3}} f_{i} d x d \xi} \quad \text { and } \quad u_{c}(t):=\int_{\mathbb{T}^{3}} u d x .
$$

Note that $\xi_{c}^{i}(t)$ and $u_{c}(t)$ are the mean velocities for the $i$-th particles and the fluid, respectively. For later use, let $\rho_{f_{i}}$ denote a local particle density:

$$
\rho_{f_{i}}(x, t):=\int_{\mathbb{R}^{3}} f_{i}(x, \xi, t) d \xi, \quad i=1,2 .
$$

In the following theorem, we find out that the system exhibits the exponential alignment between the flocking particles and the fluid.

Theorem 1.2. Let $\left(f_{1}, f_{2}, u\right)$ be classical solutions to the system (1.1)-(1.4) satisfying

(i) $\quad\left\|f_{10}\right\|_{L^{1}\left(\Omega \times \mathbb{R}^{3}\right)}=\left\|f_{20}\right\|_{L^{1}\left(\Omega \times \mathbb{R}^{3}\right)}=1$,

(ii) $\lim _{|\xi| \rightarrow \infty}|\xi|^{2}\left(f_{1}(x, \xi, t)+f_{2}(x, \xi, t)\right)=0, \quad(x, t) \in \mathbb{T}^{3} \times[0, T)$.

Suppose that $\mathcal{E}(0)<\infty$, and $\left\|\rho_{f_{i}}\right\|_{L^{\frac{3}{2}}}, i=1,2$ are sufficiently small. Then $\mathcal{E}(t)$ verifies the decay estimate:

$$
\mathcal{E}(t) \leq \mathcal{E}(0) e^{-c t}, \quad t \in[0, T] \quad \text { for some } c>0
$$

Remark 1.1. Theorem 1.2 shows that the system exhibits the exponential alignment between the particles and fluid. More precisely, since the total momentum of the system is conserved:

$$
\frac{d}{d t}\left(\int_{\mathbb{T}^{3} \times \mathbb{R}^{3}} \xi\left(f_{1}+f_{2}\right) d x d \xi+\int_{\mathbb{T}^{3}} u d x\right)=0,
$$

we obtain

$$
\begin{aligned}
\left|u_{c}(t)-\left(\xi_{c}^{1}(t)+\xi_{c}^{2}(t)\right)\right| & =2\left|u_{c}(t)-\frac{1}{2}\left(\xi_{c}^{1}(0)+\xi_{c}^{2}(0)+u_{c}(0)\right)\right| \\
& =2\left|\xi_{c}^{1}(t)+\xi_{c}^{2}(t)-\frac{1}{2}\left(\xi_{c}^{1}(0)+\xi_{c}^{2}(0)+u_{c}(0)\right)\right| .
\end{aligned}
$$


This implies that if all conditions in Theorem 1.2 hold for $T=\infty$, then the sum of particle-velocity and the fluid-velocity both converge to the half of the initial total momentum of the particles and fluid as time tends to infinity.

The paper is organized as follows. In Section 2, we prove the global existence of the unique strong solution to the nonlinear Cauchy problem of the quasi-linearized problem (1.1)-(1.4). In Section 3, we construct the approximation solutions to the original system and provide the existence of invariant sets for the approximations by employing the results in Section 2. We then derive the convergence of the approximation solutions, and this yields global existence of the unique strong solution. In Section 4, we provide the large-time behavior of classical solutions to the system (1.1)-(1.4). This result implies the system exhibits the exponential alignment between the flocking particles and fluid. Finally, Section 5 is devoted to summarize the main results and give our future work in this direction.

Notations. For a function $f(x, \xi)$, we denote by $\|f\|_{L^{p}}$ the usual $L^{p}\left(\Omega \times \mathbb{R}^{3}\right)$ norm, and if $g$ is a function of $x$ only, $\|g\|_{L^{p}}$ is the usual $L^{p}(\Omega)$-norm, otherwise specified. For simplicity, we drop $x$-dependence of differential operators $\partial_{x_{i}}(i=1,2,3), \nabla_{x}$, and $\Delta_{x}$, i.e.,

$$
\partial_{i} f:=\partial_{x_{i}} f, \quad \nabla f:=\nabla_{x} f, \quad \text { and } \quad \Delta f:=\Delta_{x} f .
$$

\section{Global existence for the linearized system}

In this section, we first linearize the system (1.1) with respect to the fluid velocity $u$ in the drag forces, and show the existence for the linearized system. We also provide uniform boundness of the unique solution.

Consider the linearized system:

$$
\begin{aligned}
& \partial_{t} f_{1}+\xi \cdot \nabla f_{1}+\nabla_{\xi} \cdot\left(\left(F_{a}^{1}\left[f_{1}\right]+F_{d}[v]\right) f_{1}\right)=0, \quad(x, \xi) \in \Omega \times \mathbb{R}^{3}, \quad t>0, \\
& \partial_{t} f_{2}+\xi \cdot \nabla f_{2}+\nabla_{\xi} \cdot\left(\left(F_{a}^{2}\left[f_{2}\right]+F_{d}[v]\right) f_{2}\right)=0, \quad(x, \xi) \in \Omega \times \mathbb{R}^{3}, \quad t>0, \\
& \partial_{t} u+v \cdot \nabla u+\nabla p-\Delta u \\
& \quad=-\int_{\mathbb{R}^{3}} F_{d}[v]\left(f_{1}+f_{2}\right) d \xi, \quad \nabla \cdot u=0, \quad x \in \Omega, t>0,
\end{aligned}
$$

where $v$ is a known vector field. For this system, we present the existence, smallness, and regularity results as follows. 
Theorem 2.1. Let $T \in(0, \infty)$. Suppose that the initial data $\left(f_{10}, f_{20}, u\right)$ satisfy the smallness, regularity and compactly supported conditions:

(i) $\left\|f_{10}\right\|_{W^{1, \infty}}+\left\|f_{20}\right\|_{W^{1, \infty}}+\left\|u_{0}\right\|_{H^{2}}<\varepsilon$,

(ii) $f_{10}$ and $f_{20}$ have a compact support in position and velocity.

Furthermore, $v$ satisfies the smallness and regularity conditions:

$\|v\|_{\mathcal{C}\left([0, T] ; H^{2}\right)}+\|v\|_{L^{2}\left(0, T ; H^{3}\right)} \leq \varepsilon^{\alpha} \quad$ and $\quad\left\|v_{t}\right\|_{\mathcal{C}\left([0, T] ; L^{2}\right)}+\left\|v_{t}\right\|_{L^{2}\left(0, T ; H^{1}\right)} \leq \varepsilon^{\alpha^{-}}$,

where $\alpha^{-}:=\alpha-\varepsilon_{1}$ for sufficiently small $\varepsilon_{1}>0$. Then there exists a unique strong solution $\left(f_{1}, f_{2}, u\right)$ to the Cauchy problem (2.1), (1.2)-(1.4) such that

(i) $\left\|f_{1}\right\|_{L^{\infty}\left(0, T ; W^{1, \infty}\right)},\left\|f_{2}\right\|_{L^{\infty}\left(0, T ; W^{1, \infty}\right)} \leq \varepsilon^{\beta}$,

(ii) $\|u\|_{\mathcal{C}\left([0, T] ; H^{2}\right)}+\|u\|_{L^{2}\left(0, T ; H^{3}\right)} \leq \varepsilon^{\alpha} \quad$ and $\left\|u_{t}\right\|_{\mathcal{C}\left([0, T] ; L^{2}\right)}+\left\|u_{t}\right\|_{L^{2}\left(0, T ; H^{1}\right)} \leq \varepsilon^{\alpha^{-}}$.

Here $\alpha$ and $\beta$ are positive numbers with $1>\beta>\alpha>0$, and $\varepsilon>0$ is a sufficiently small constant such that $\varepsilon \approx e^{-\mathcal{O}(1) T}$.

We first solve the Vlasov-type equations in (2.1). We notice that the theory of local existence and regularity of a unique strong solution to the system $(2.1)_{1}$ and $(2.1)_{2}$ have been well-known when $v \in \mathcal{C}\left([0, T] ; H^{2}\right)$. For the estimate of uniform bound of $f_{i}$ in (2.4), we need to control the propagation of support of $f_{i}$ in velocity. For this, we introduce new notations here. Let $\Sigma_{x}^{i}(t)$ and $\Sigma_{\xi}^{i}(t)$ be the $x, \xi$-projections of $\operatorname{supp} f_{i}(\cdot, \cdot, t)$, respectively, $i=1,2$ :

$$
\begin{aligned}
& \Sigma_{x}^{i}(t):=\left\{x \in \Omega \quad: \exists(x, \xi) \in \Omega \times \mathbb{R}^{3} \quad \text { such that } \quad f_{i}(x, \xi, t) \neq 0\right\} \\
& \Sigma_{\xi}^{i}(t):=\left\{\xi \in \mathbb{R}^{3} \quad: \exists(x, \xi) \in \Omega \times \mathbb{R}^{3} \quad \text { such that } \quad f_{i}(x, \xi, t) \neq 0\right\} .
\end{aligned}
$$

Then we define $R_{x}^{i}(t)$ and $R_{\xi}^{i}(t)$ by

$$
R_{x}^{i}(t):=\max _{x \in \Sigma_{x}^{i}(t)}|x|, \quad R_{\xi}^{i}(t):=\max _{\xi \in \Sigma_{\xi}^{i}(t)}|\xi|
$$

We now present the estimates for the support of $f_{i}$ in position and velocity.

Lemma 2.1. For $i=1,2$, let $\left(X_{i}(s), V_{i}(s)\right):=\left(X_{i}(s ; 0, x, \xi), V_{i}(s ; 0, x, \xi)\right)$ 
be the forward particle trajectories solving the following ODEs:

$$
\begin{aligned}
\frac{d X_{i}(s)}{d s}= & V_{i}(s) \\
\frac{d V_{i}(s)}{d s}= & \int_{\Omega \times \mathbb{R}^{3}} \psi_{i}\left(X_{i}(s), y\right)\left(\xi_{*}-V_{i}(s)\right) f_{i}\left(y, \xi_{*}, s\right) d y d \xi_{*} \\
& +v\left(X_{i}(s), s\right)-V_{i}(s)
\end{aligned}
$$

with initial data $\left(X_{i}(0), V_{i}(0)\right)=(x, \xi)$. Then we have

$$
\begin{gathered}
\left|X_{i}(t)\right| \leq\left|R_{x}^{i}(0)\right|+\left(\left|R_{\xi}^{i}(0)\right|+\|v\|_{L^{1}\left(0, T ; L^{\infty}\right)}\right) T, \\
\left|V_{i}(t)\right| \leq\left|R_{\xi}^{i}(0)\right|+\|v\|_{L^{1}\left(0, T ; L^{\infty}\right)}, \quad i=1,2 .
\end{gathered}
$$

Proof. From the regularity results for $f_{i}$ and $v$, we find that $R_{\xi}^{i}(t)$ is a Lipschitz function and differentiable with respect to time $t$ almost everywhere. This enable us to choose $V_{i}(t)$ so that $\frac{d}{d t} R_{\xi}^{i}(t)$ exists and $R_{\xi}^{i}(t)=\left|V_{i}(t)\right|$. Then we obtain

$$
\begin{aligned}
& \frac{1}{2} \frac{d}{d t}\left(R_{\xi}^{i}(t)\right)^{2}=\frac{1}{2} \frac{d}{d t}\left|V_{i}(t)\right|^{2}=V_{i}(t) \cdot \frac{d V_{i}(t)}{d t} \\
& \quad=\int_{\Omega \times \mathbb{R}^{3}} \psi_{i}\left(X_{i}(t), y\right)\left(\xi_{*}-V_{i}(t)\right) \cdot V_{i}(t) f_{i}\left(y, \xi_{*}, t\right) d y d \xi_{*} \\
& \quad+v\left(X_{i}(t), t\right) \cdot V_{i}(t)-\left|V_{i}(t)\right|^{2} \\
& \quad \leq|v(t)|_{L^{\infty}}\left|V_{i}(t)\right|-\left|V_{i}(t)\right|^{2}
\end{aligned}
$$

where we used

$$
\left(\xi_{*}-V_{i}(t)\right) \cdot V_{i}(t) \leq 0 \quad \text { for } \quad \xi_{*} \in \Sigma_{\xi}^{i}(t) .
$$

This implies

$$
\left|R_{\xi}^{i}(t)\right| \leq\left|R_{\xi}^{i}(0)\right|+\|v\|_{L^{1}\left(0, T ; L^{\infty}\right)},
$$

and

$$
\left|R_{x}^{i}(t)\right| \leq\left|R_{x}^{i}(0)\right|+\left|R_{\xi}^{i}(0)\right| T+\|v\|_{L^{1}\left(0, T ; L^{\infty}\right)} T .
$$

For notational simplicity, we set

$$
R_{x}^{i, \infty}:=\sup _{0 \leq t \leq T} R_{x}^{i}(t), \quad R_{\xi}^{i, \infty}:=\sup _{0 \leq t \leq T} R_{\xi}^{i}(t), \quad \pi\left(R_{x}^{i, \infty}\right):=\operatorname{vol}\left(B_{R_{x}^{i, \infty}}\right),
$$

and

$$
\pi\left(R_{\xi}^{i, \infty}\right):=\operatorname{vol}\left(B_{R_{\xi}^{i, \infty}}\right), \quad \text { for } \quad i=1,2
$$


We now establish the estimate of uniform bound of $f_{i}$ in $W^{1, \infty}$-norm. For this, we first present the following simple calculations without proof.

Lemma 2.2. For $i=1,2$, let $f_{i}$ be classical solutions to the system (2.1), (1.4) with compactly supported initial data $f_{i 0}$ in velocity. Suppose that $v$ satisfies (2.3). Then the following estimates hold.

$$
\begin{gathered}
(i)-\nabla_{\xi} \cdot\left(F_{a}^{i}\left[f_{i}\right]+F_{d}[v]\right)= \\
\leq \int_{\Omega \times \mathbb{R}^{3}} \psi_{i}(x, y) f_{i}\left(y, \xi_{*}\right) d y d \xi_{*}-3 \\
\leq 3\left\|\psi_{i}\right\|_{L^{\infty}} M_{i 0}, \\
(i i)-\partial_{j}\left(F_{a}^{i}\left[f_{i}\right]+F_{d}[v]\right)=-\int_{\Omega \times \mathbb{R}^{3}} \partial_{j} \psi_{i}(x, y)\left(\xi_{*}-\xi\right) f_{i}\left(y, \xi_{*}\right) d y d \xi_{*}-\partial_{j} v \\
\leq 2 R_{\xi}^{i, \infty}\left\|\partial_{j} \psi_{i}\right\|_{L^{\infty}} M_{i 0}+\left\|\partial_{j} v\right\|_{L^{\infty}}, \\
(i i i)-\nabla_{\xi} \cdot \partial_{j}\left(F_{a}^{i}\left[f_{i}\right]+F_{d}[v]\right)=3 \int_{\Omega \times \mathbb{R}^{3}} \partial_{j} \psi_{i}(x, y) f_{i}\left(y, \xi_{*}\right) d y d \xi_{*} \\
\leq 3\left\|\partial_{j} \psi_{i}\right\|_{L^{\infty}} M_{i 0},
\end{gathered}
$$

where $M_{i 0}:=\int_{\Omega \times \mathbb{R}^{3}} f_{i 0}(x, \xi) d x d \xi<\infty$.

Lemma 2.3. For $i=1,2$, let $f_{i}$ be classical solutions to the system (2.1), (1.4) with the initial data $f_{i 0}$ satisfying (2.2). If $v$ satisfies the smallness conditions (2.3), then we have

$$
\left\|f_{i}\right\|_{L^{\infty}\left(0, T ; W^{1, \infty}\right)}<\varepsilon^{\beta}, \quad i=1,2 .
$$

Proof. Similarly as the arguments in [2], we introduce a nonlinear operator $\mathcal{N}_{i}:=\partial_{t}+\xi \cdot \nabla+\left(F_{a}^{i}\left[f_{i}\right]+F_{d}[v]\right) \cdot \nabla_{\xi}$ which is associated with $f_{i}$. Then we employ the estimates in Lemma 2.2 to obtain

$$
\begin{aligned}
\mathcal{N}_{i}\left(f_{i}\right)= & -\nabla_{\xi} \cdot\left(F_{a}^{i}\left[f_{i}\right]+F_{d}[v]\right) f_{i} \leq 3\left\|\psi_{i}\right\|_{L^{\infty}} M_{i 0} \\
\mathcal{N}_{i}\left(\partial_{j} f_{i}\right)= & -\partial_{j}\left(F_{a}^{i}\left[f_{i}\right]+F_{d}[v]\right) \cdot \nabla_{\xi} f_{i}-\left(\nabla_{\xi} \cdot \partial_{j}\left(F_{a}^{i}\left[f_{i}\right]+F_{d}[v]\right)\right) f_{i} \\
& -\left(\nabla \cdot\left(F_{a}^{i}\left[f_{i}\right]+F_{d}[v]\right)\right) \partial_{j} f_{i} \\
\leq & \left(2 R_{\xi}^{i}(t)\left\|\psi_{i}\right\|_{L^{\infty}} M_{i 0}+\left\|\partial_{j} v\right\|_{L^{\infty}}\right)\left|\nabla_{\xi} f_{i}\right|+3\left\|\partial_{j} \psi_{i}\right\|_{L^{\infty}} M_{i 0}\left|f_{i}\right| \\
& +3\left(\left\|\psi_{i}\right\|_{L^{\infty}} M_{i 0}+1\right)\left|\partial_{j} f_{i}\right|, \\
\mathcal{N}_{i}\left(\partial_{\xi_{j}}\left(f_{i}\right)\right)= & -\partial_{j} f_{i}-\partial_{\xi_{j}}\left(F_{a}^{i}\left[f_{i}\right]+F_{d}[v]\right) \cdot \nabla_{\xi} f_{i} \\
& -\left(\nabla_{\xi} \cdot\left(F_{a}^{i}\left[f_{i}\right]+F_{d}[v]\right)\right) \partial_{\xi_{j}} f_{i} \\
\leq & \left|\partial_{j} f_{i}\right|+\left(\left\|\psi_{i}\right\|_{L^{\infty}} M_{i 0}+1\right)\left|\nabla_{\xi} f_{i}\right|+3\left(\left\|\psi_{i}\right\|_{L^{\infty}} M_{i 0}+1\right)\left|\nabla_{\xi_{j}} f_{i}\right|,
\end{aligned}
$$


for $i=1,2$. We set $\mathcal{F}_{i}(t)$ measuring $f$ in $W^{1, \infty}$-norm:

$$
\mathcal{F}_{i}(t):=\sum_{0 \leq|\alpha|+|\beta| \leq 1}\left\|\nabla^{\alpha} \nabla_{\xi}^{\beta} f_{i}(t)\right\|_{L^{\infty}} .
$$

Then using the previous estimates, we obtain

$$
\frac{d \mathcal{F}_{i}(t)}{d t} \leq C\left(1+\|\nabla v\|_{L^{\infty}}\right) \mathcal{F}_{i}(t), \quad t \in(0, T) .
$$

This yields

$$
\mathcal{F}_{i}(t) \leq \mathcal{F}_{i}(0) \exp \left(C\left(T+\sqrt{T}\|v\|_{L^{2}\left(0, T ; H^{3}\right)}\right)\right), \quad t \in(0, T) .
$$

Since $1>\beta>\alpha$, for the sufficiently small $\varepsilon \ll 1$, we obtain

$$
\left\|f_{i}\right\|_{W^{1, \infty}} \leq \varepsilon \exp \left(C\left(T+\sqrt{T} \varepsilon^{\alpha}\right)\right)<\varepsilon^{\beta} .
$$

This yields that for $i=1,2$,

$$
\sup _{0 \leq t \leq T}\left\|f_{i}\right\|_{W^{1, \infty}} \leq \varepsilon^{\beta} .
$$

Remark 2.1. From the structure of Vlasov-type equations, one can easily check that

$$
f_{i} \in W^{1, \infty}\left(\Omega \times \mathbb{R}^{3} \times(0, T)\right), \quad i=1,2 .
$$

Proof of Theorem 2.1. We first notice that $f_{1}, f_{2} \in W^{1, \infty}\left(\Omega \times \mathbb{R}^{3} \times(0, T)\right)$, $v \in \mathcal{C}\left([0, T] ; H^{2}\right) \cap L^{2}\left(0, T ; H^{3}\right)$, and $v_{t} \in \mathcal{C}\left([0, T] ; L^{2}\right) \cap L^{2}\left(0, T ; H^{1}\right)$. Thus the existence and regularity of the unique solution $u$ can be proved by a standard method. Then we obtain the estimates of uniform bounds in (2.4). This proof is a rather lengthy, so we divide it into five steps.

- Step A.- Estimate of $\|u\|_{L^{\infty}\left(0, T ; L^{2}\right)}+\|\nabla u\|_{L^{2}\left(0, T ; L^{2}\right)}$ : It follows from $(2.1)_{3}$ that

$$
\begin{aligned}
\frac{1}{2} \frac{d}{d t}\|u\|_{L^{2}}^{2}+\|\nabla u\|_{L^{2}}^{2}= & -\int_{\Omega}(v \cdot \nabla u) \cdot u d x \\
& -\int_{\Omega \times \mathbb{R}^{3}}(v \cdot u-u \cdot \xi)\left(f_{1}+f_{2}\right) d x d \xi \\
= & : I_{1}+I_{2},
\end{aligned}
$$


where $I_{j}, j=1,2$ are estimated as follows.

$$
\begin{aligned}
I_{1} & =\frac{1}{2} \int_{\Omega}(\nabla \cdot v)|u|^{2} d x \leq \frac{1}{2}\|\nabla v\|_{L^{3}}\|u\|_{L^{6}}\|u\|_{L^{2}} \leq \frac{1}{2}\|\nabla v\|_{H^{1}}\|\nabla u\|_{L^{2}}\|u\|_{L^{2}} \\
& \leq \varepsilon^{\alpha}\|\nabla u\|_{L^{2}}\|u\|_{L^{2}}
\end{aligned}
$$

and

$$
\begin{aligned}
I_{2} \leq & \|v\|_{L^{2}}\|u\|_{L^{2}}\left\|\int_{\mathbb{R}^{3}} f_{1}+f_{2} d \xi\right\|_{L^{\infty}}+\|u\|_{L^{2}}\left\|\int_{\mathbb{R}^{3}} \xi\left(f_{1}+f_{2}\right) d \xi\right\|_{L^{2}} \\
\leq & \left(\sum_{j=1}^{2}\left\|f_{i}\right\|_{L^{\infty}} \pi\left(R_{\xi}^{i, \infty}\right)\right)\|v\|_{L^{2}}\|u\|_{L^{2}} \\
& +\left(\sum_{j=1}^{2}\left\|f_{i}\right\|_{L^{\infty}} R_{\xi}^{i, \infty} \pi\left(R_{\xi}^{i, \infty}\right) \pi\left(R_{x}^{i, \infty}\right)\right)\|u\|_{L^{2}} .
\end{aligned}
$$

Here we used

$$
\begin{aligned}
& \left\|\int_{\mathbb{R}^{3}} f_{i} d \xi\right\|_{L^{\infty}} \leq\left\|f_{i}\right\|_{L^{\infty}} \pi\left(R_{\xi}^{i, \infty}\right) \text { and } \\
& \left\|\int_{\mathbb{R}^{3}} \xi f_{i} d \xi\right\|_{L^{2}} \leq\left\|f_{i}\right\|_{L^{\infty}} R_{\xi}^{i, \infty} \pi\left(R_{\xi}^{i, \infty}\right) \pi\left(R_{x}^{i, \infty}\right),
\end{aligned}
$$

for $i=1,2$. Combining (2.5) and (2.6), we get

$$
\begin{aligned}
\frac{1}{2} \frac{d}{d t}\|u\|_{L^{2}}^{2}+\|\nabla u\|_{L^{2}}^{2} & \leq C\|u\|_{L^{2}}\left(\varepsilon^{\alpha}\|\nabla u\|_{L^{2}}+\varepsilon^{\alpha+\beta}+\varepsilon^{\beta}\right) \\
& \leq \frac{1}{2}\|u\|_{L^{2}}^{2}+\frac{1}{2}\|\nabla u\|_{L^{2}}^{2}+C \varepsilon^{2 \beta}
\end{aligned}
$$

Applying the Gronwall's inequality for $\|u\|_{L^{2}}^{2}$ and integrating over $[0, T]$, we obtain

$$
\sup _{0 \leq t \leq T}\|u\|_{L^{2}}^{2}+\int_{0}^{T}\|\nabla u\|_{L^{2}}^{2} d t \leq C\left(\left\|u_{0}\right\|_{L^{2}}^{2}+\varepsilon^{2 \beta}\right) e^{T} \leq C\left(\varepsilon^{2}+\varepsilon^{2 \beta}\right) e^{T} \leq \varepsilon^{2 \alpha} .
$$

- Step B.- Estimate of $\|\nabla u\|_{L^{\infty}\left(0, T ; L^{2}\right)}+\left\|\nabla^{2} u\right\|_{L^{2}\left(0, T ; L^{2}\right)}$ : We differentiate 
$(2.1)_{3}$ with respect to $x$ and integrate over $\Omega$ to find

$$
\begin{aligned}
\frac{1}{2} \frac{d}{d t} & \|\nabla u\|_{L^{2}}^{2}+\left\|\nabla^{2} u\right\|_{L^{2}}^{2} \\
& =-\int_{\Omega} \nabla(v \cdot \nabla u) \cdot \nabla u d x-\int_{\Omega \times \mathbb{R}^{3}} \nabla\left(F_{d}[v]\left(f_{1}+f_{2}\right)\right) \cdot \nabla u d x d \xi \\
& =: J_{1}+J_{2} .
\end{aligned}
$$

We estimate $J_{j}, j=1,2$, as follows:

$$
\begin{aligned}
J_{1} & \leq \int_{\Omega}\left|\nabla v \left\|\left.\nabla u\right|^{2} d x+\int_{\Omega}\left|v\left\|\nabla^{2} u\right\| \nabla u\right| d x\right.\right. \\
& \leq\|\nabla v\|_{L^{3}}\|\nabla u\|_{L^{6}}\|\nabla u\|_{L^{2}}+\|v\|_{L^{\infty}}\left\|\nabla^{2} u\right\|_{L^{2}}\|\nabla u\|_{L^{2}} \\
& \leq C \varepsilon^{\alpha}\left\|\nabla^{2} u\right\|_{L^{2}}\|\nabla u\|_{L^{2}}
\end{aligned}
$$

and

$$
\begin{aligned}
J_{2} \leq & \int_{\Omega \times \mathbb{R}^{3}}\left|\nabla v\|\nabla u\| f_{1}+f_{2}\right| d x d \xi+\int_{\Omega \times \mathbb{R}^{3}}\left|v-\xi\|\nabla u\| \nabla f_{1}+\nabla f_{2}\right| d x d \xi \\
\leq & C\left(\sum_{i=1}^{2}\left\|f_{i}\right\|_{L^{\infty}} \pi\left(R_{\xi}^{i, \infty}\right)\right)\|\nabla v\|_{L^{2}}\|\nabla u\|_{L^{2}} \\
& +C\left(\sum_{i=1}^{2}\left\|\nabla f_{i}\right\|_{L^{\infty}} \pi\left(R_{\xi}^{i, \infty}\right)\right)\|v\|_{L^{2}}\|\nabla u\|_{L^{2}} \\
& +C\left(\sum_{i=1}^{2}\left\|\nabla f_{i}\right\|_{L^{\infty}} R_{\xi}^{i, \infty} \pi\left(R_{\xi}^{i, \infty}\right) \pi\left(R_{x}^{i, \infty}\right)^{\frac{1}{2}}\right)\|\nabla u\|_{L^{2}} \\
\leq & C \varepsilon^{\beta}\|\nabla u\|_{L^{2}} .
\end{aligned}
$$

Then we obtain

$$
\begin{aligned}
\frac{1}{2} \frac{d}{d t}\|\nabla u\|_{L^{2}}^{2}+\left\|\nabla^{2} u\right\|_{L^{2}}^{2} & \leq C \varepsilon^{\alpha}\left\|\nabla^{2} u\right\|_{L^{2}}\|\nabla u\|_{L^{2}}+C \varepsilon^{\beta}\|\nabla u\|_{L^{2}} \\
& \leq \frac{1}{2}\|\nabla u\|_{L^{2}}^{2}+\frac{1}{2}\left\|\nabla^{2} u\right\|_{L^{2}}^{2}+C \varepsilon^{2 \beta},
\end{aligned}
$$

and this implies that

$$
\begin{aligned}
\sup _{0 \leq t \leq T}\|\nabla u\|_{L^{2}}^{2}+\int_{0}^{T}\left\|\nabla^{2} u\right\|_{L^{2}}^{2} d t & \leq C\left(\left\|\nabla u_{0}\right\|_{L^{2}}^{2}+\varepsilon^{2 \beta}\right) e^{T} \\
& \leq C\left(\varepsilon^{2}+\varepsilon^{2 \beta}\right) e^{T} \leq \varepsilon^{2 \alpha} .
\end{aligned}
$$


- Step C.- Estimate of $\left\|\nabla^{2} u\right\|_{L^{\infty}\left(0, T ; L^{2}\right)}+\left\|\nabla^{3} u\right\|_{L^{2}\left(0, T ; L^{2}\right)}$ : We take the spatial differential operator $\nabla^{2}$ to the system $(2.1)_{3}$ and integrate over $\Omega$ to the following equality.

$$
\begin{aligned}
& \frac{1}{2} \frac{d}{d t}\left\|\nabla^{2} u\right\|_{L^{2}}^{2}+\left\|\nabla^{3} u\right\|_{L^{2}}^{2} \\
& \quad=-\int_{\Omega} \nabla^{2}(v \cdot \nabla u): \nabla^{2} u d x-\int_{\Omega \times \mathbb{R}^{3}} \nabla^{2}\left(F_{d}[v]\left(f_{1}+f_{2}\right)\right): \nabla^{2} u d x d \xi \\
& \quad=: K_{1}+K_{2},
\end{aligned}
$$

where $K_{j}, j=1,2$ are estimated as

$$
\begin{aligned}
K_{1} \leq & C \int_{\Omega}\left|\nabla^{2} v\|\nabla u\| \nabla^{2} u\right| d x+C \int_{\Omega}\left|\nabla v\left\|\nabla^{2} u\right\| \nabla^{2} u\right| d x \\
& +C \int_{\Omega}\left|v\left\|\nabla^{3} u\right\| \nabla^{2} u\right| d x \\
\leq & C\left\|\nabla^{2} v\right\|_{L^{2}}\|\nabla u\|_{L^{3}}\left\|\nabla^{2} u\right\|_{L^{6}}+C\|\nabla v\|_{L^{3}}\left\|\nabla^{2} u\right\|_{L^{6}}\left\|\nabla^{2} u\right\|_{L^{2}} \\
& +C\|v\|_{L^{\infty}}\left\|\nabla^{3} u\right\|_{L^{2}}\left\|\nabla^{2} u\right\|_{L^{2}} \\
\leq & C \varepsilon^{\alpha}\left(\|\nabla u\|_{L^{2}}^{\frac{1}{2}}+\left\|\nabla^{2} u\right\|_{L^{2}}^{\frac{1}{2}}\right)\left\|\nabla^{2} u\right\|_{L^{2}}^{\frac{1}{2}}\left\|\nabla^{3} u\right\|_{L^{2}} \\
\leq & \frac{1}{4}\left\|\nabla^{2} u\right\|_{L^{2}}^{2}+\frac{1}{4}\left\|\nabla^{3} u\right\|_{L^{2}}^{2}+C \varepsilon^{6 \alpha},
\end{aligned}
$$

and

$$
\begin{aligned}
K_{2} \leq & C \int_{\Omega \times \mathbb{R}^{3}}\left|\nabla^{2} v\left\|f_{1}+f_{2}\right\| \nabla^{2} u\right| d x d \xi+\int_{\Omega \times \mathbb{R}^{3}}\left|\nabla v\left\|\nabla f_{1}+\nabla f_{2}\right\| \nabla^{2} u\right| d x d \xi \\
& +\int_{\Omega \times \mathbb{R}^{3}}\left|v-\xi\left\|\nabla f_{1}+\nabla f_{2}\right\| \nabla^{3} u\right| d x d \xi \\
\leq & C\left(\sum_{i=1}^{2}\left\|f_{i}\right\|_{L^{\infty}} \pi\left(R_{\xi}^{i, \infty}\right)\right)\left\|\nabla^{2} v\right\|_{L^{2}}\left\|\nabla^{2} u\right\|_{L^{2}} \\
& +C\left(\sum_{i=1}^{2}\left\|\nabla f_{i}\right\|_{L^{\infty}} \pi\left(R_{\xi}^{i, \infty}\right)\right)\|\nabla v\|_{L^{2}}\left\|\nabla^{2} u\right\|_{L^{2}} \\
& +C\left(\sum_{i=1}^{2}\left\|\nabla f_{i}\right\|_{L^{\infty}} \pi\left(R_{\xi}^{i, \infty}\right)\left(\|v\|_{L^{2}}+R_{\xi}^{i, \infty}\left(\pi\left(R_{x}^{i, \infty}\right)\right)^{\frac{1}{2}}\right)\right)\left\|\nabla^{3} u\right\|_{L^{2}} \\
\leq & C \varepsilon^{\alpha+\beta}\left\|\nabla^{2} u\right\|_{L^{2}}+C \varepsilon^{\beta}\left(1+\varepsilon^{\alpha}\right)\left\|\nabla^{3} u\right\|_{L^{2}} \\
\leq & \frac{1}{4}\left\|\nabla^{2} u\right\|_{L^{2}}^{2}+\frac{1}{4}\left\|\nabla^{3} u\right\|_{L^{2}}^{2}+C \varepsilon^{2 \beta} .
\end{aligned}
$$


This yields

$$
\frac{1}{2} \frac{d}{d t}\left\|\nabla^{2} u\right\|_{L^{2}}^{2}+\left\|\nabla^{3} u\right\|_{L^{2}}^{2} \leq \frac{1}{2}\left\|\nabla^{2} u\right\|_{L^{2}}^{2}+\left\|\nabla^{3} u\right\|_{L^{2}}^{2}+C \varepsilon^{6 \alpha}+C \varepsilon^{2 \beta}
$$

and

$$
\begin{aligned}
\sup _{0 \leq t \leq T}\left\|\nabla^{2} u\right\|_{L^{2}}^{2}+\int_{0}^{T}\left\|\nabla^{3} u\right\|_{L^{2}}^{2} d t & \leq C\left(\left\|\nabla^{2} u_{0}\right\|_{L^{2}}^{2}+\varepsilon^{2 \beta}+\varepsilon^{4 \alpha}\right) e^{T} \\
& \leq C\left(\varepsilon^{2}+\varepsilon^{2 \beta}+\varepsilon^{4 \alpha}\right) e^{T} \\
& \leq \varepsilon^{2 \alpha}
\end{aligned}
$$

Combining the estimates (2.7), (2.8), and (2.9), we arrive at

$$
\|u\|_{L^{\infty}\left(0, T ; H^{2}\right)}+\|\nabla u\|_{L^{2}\left(0, T ; H^{2}\right)} \leq \varepsilon^{\alpha} .
$$

- Step D.- Estimate of $\left\|u_{t}\right\|_{L^{\infty}\left(0, T ; L^{2}\right)}+\left\|u_{t}\right\|_{L^{2}\left(0, T ; H^{1}\right)}$ : We first multiply $(2.1)_{3}$ by $\partial_{t} u$ and integrate over $\Omega$ to find

$$
\begin{aligned}
\frac{1}{2} \int_{\Omega}\left|u_{t}\right|^{2} d x+\frac{d}{d t} \int_{\Omega}|\nabla u|^{2} d x \leq & \int_{\Omega}|v|^{2}|\nabla u|^{2} d x \\
& +\int_{\Omega}\left(\int_{\mathbb{R}^{3}}|v-\xi|\left(f_{1}+f_{2}\right) d \xi\right)^{2} d x \\
\leq & C\left(\varepsilon^{4 \alpha}+\varepsilon^{2 \beta}\left(1+\varepsilon^{2 \alpha}\right)\right) .
\end{aligned}
$$

Then we get

$$
\int_{0}^{T} \int_{\Omega}\left|u_{t}\right|^{2} d x d t \leq \int_{\Omega}\left|\nabla u_{0}\right|^{2} d x+C\left(\varepsilon^{4 \alpha}+\varepsilon^{2 \beta}\right) T \leq \varepsilon^{2 \alpha}
$$

In order to derive the higher regularity estimates, we next differentiate $(2.1)_{3}$ with respect to $t$ to obtain

$$
u_{t t}+v \cdot \nabla u_{t}+\nabla p_{t}-\Delta u_{t}=-v_{t} \cdot \nabla u-\int_{\mathbb{R}^{3}} v_{t}\left(f_{1}+f_{2}\right) d \xi-\int_{\mathbb{R}^{3}}(v-\xi)\left(f_{1}+f_{2}\right)_{t} d \xi
$$


Multiplying (2.12) by $u_{t}$ and integrating over $\Omega$, one can obtain

$$
\begin{aligned}
\frac{1}{2} \frac{d}{d t} & \int_{\Omega}\left|u_{t}\right|^{2} d x+\int_{\Omega}\left|\nabla u_{t}\right|^{2} d x \\
\leq & \left\|u_{t}\right\|_{L^{2}}\left\|v_{t}\right\|_{L^{2}}\|\nabla u\|_{L^{\infty}}+\left\|u_{t}\right\|_{L^{2}}\|v\|_{L^{\infty}}\left\|\nabla u_{t}\right\|_{L^{2}} \\
& +\left\|u_{t}\right\|_{L^{2}}\left\|v_{t}\right\|_{L^{2}}\left(\left\|f_{1}\right\|_{L^{\infty}}+\left\|f_{2}\right\|_{L^{\infty}}\right) \\
& +C\left\|\nabla u_{t}\right\|_{L^{2}}\left(\left\|f_{1}\right\|_{L^{\infty}}+\left\|f_{2}\right\|_{L^{\infty}}\right)\left(\|v\|_{L^{2}}+1\right) \\
& +C\left\|u_{t}\right\|_{L^{2}}\|\nabla v\|_{L^{2}}\left(\left\|f_{1}\right\|_{L^{\infty}}+\left\|f_{2}\right\|_{L^{\infty}}\right) \\
& +C\left\|u_{t}\right\|_{L^{2}}\left(\left\|f_{1}\right\|_{L^{\infty}}+\left\|f_{2}\right\|_{L^{\infty}}\right) \\
& +C\left\|u_{t}\right\|_{L^{2}}\left(\left\|f_{1}\right\|_{L^{\infty}}+\left\|f_{2}\right\|_{L^{\infty}}\right)\left(\|v\|_{L^{2}}+1\right) \\
\leq & C\left\|u_{t}\right\|_{L^{2}}^{2}+\frac{1}{2} \int_{\Omega}\left|\nabla u_{t}\right|^{2} d x+C \varepsilon^{2 \alpha}\|\nabla u\|_{L^{\infty}}^{2}+C \varepsilon^{2 \beta} .
\end{aligned}
$$

This implies

$$
\int_{\Omega}\left|u_{t}\right|^{2}(t) d x+\int_{s}^{t} \int_{\Omega}\left|\nabla u_{t}\right|^{2} d x d \tau \leq \int_{\Omega}\left|u_{t}\right|^{2}(s) d x e^{C T}+C\left(\varepsilon^{4 \alpha}+\varepsilon^{2 \beta}\right) e^{C T} .
$$

On the other hand, similarly as in (2.11), one can have that

$$
\begin{aligned}
\int_{\Omega}\left|u_{t}\right|^{2} d x= & \int_{\Omega}\left(\int_{\mathbb{R}^{3}}(\xi-v)\left(f_{1}+f_{2}\right) d \xi-v \cdot \nabla u+\Delta u\right) \cdot u_{t} d x \\
\leq & C \int_{\Omega}\left(\int_{\mathbb{R}^{3}}(\xi-v)\left(f_{1}+f_{2}\right)\right)^{2} d x+C \int_{\Omega}|v|^{2}|\nabla u|^{2} d x \\
& +C \int_{\Omega}\left|\nabla^{2} u\right|^{2} d x \\
\leq & C\left(\varepsilon^{2 \alpha}+\varepsilon^{2 \beta}\right) \leq C \varepsilon^{2 \alpha}
\end{aligned}
$$

Hence we combine (2.13) and (2.14) to have

$$
\begin{aligned}
\sup _{0 \leq t \leq T} \int_{\Omega}\left|u_{t}\right|^{2} d x+\int_{0}^{T} \int_{\Omega}\left|\nabla u_{t}\right|^{2} d x d t & \leq \limsup _{s \rightarrow 0} \int_{\Omega}\left|u_{t}\right|^{2}(s) d x e^{C T} \\
& +C\left(\varepsilon^{4 \alpha}+\varepsilon^{2 \beta}\right) \\
\leq & C \varepsilon^{2 \alpha} \leq \varepsilon^{2 \alpha^{-}}
\end{aligned}
$$

and this concludes

$$
\left\|u_{t}\right\|_{L^{\infty}\left(0, T ; L^{2}\right)}+\left\|u_{t}\right\|_{L^{2}\left(0, T ; H^{1}\right)} \leq \varepsilon^{2 \alpha^{-}} .
$$


- Step E.- $\left(u, u_{t}\right) \in \mathcal{C}\left([0, T] ; H^{2}(\Omega)\right) \times \mathcal{C}\left([0, T] ; L^{2}(\Omega)\right)$ : From Step C and $\mathrm{D}$, we find that

$$
u \in L^{2}\left(0, T ; H^{3}(\Omega)\right) \quad \text { and } \quad u_{t} \in L^{2}\left(0, T ; H^{1}(\Omega)\right) .
$$

Thus, by using a standard Sobolev embedding, we obtain $u \in \mathcal{C}\left([0, T] ; H^{2}(\Omega)\right)$. Then by this continuity of $u$ in $H^{2}(\Omega)$, we deduce from the momentum equations that $u_{t} \in \mathcal{C}\left([0, T] ; L^{2}(\Omega)\right)$. This completes the proof.

\section{Proof of Theorem 1.1}

In this section, we give the proof of our first main result. For this, we consider the following approximated sequences:

$$
\begin{aligned}
& \partial_{t} f_{1}^{n+1}+\xi \cdot \nabla f_{1}^{n+1}+\nabla_{\xi} \cdot\left(\left(F_{a}^{1}\left[f_{1}^{n+1}\right]+F_{d}\left[u^{n}\right]\right) f_{1}^{n+1}\right)=0, \\
& \quad(x, \xi) \in \Omega \times \mathbb{R}^{3}, \quad t>0, \\
& \partial_{t} f_{2}^{n+1}+\xi \cdot \nabla f_{2}^{n+1}+\nabla_{\xi} \cdot\left(\left(F_{a}^{2}\left[f_{2}^{n+1}\right]+F_{d}\left[u^{n}\right]\right) f_{2}^{n+1}\right)=0, \\
& \quad(x, \xi) \in \Omega \times \mathbb{R}^{3}, \quad t>0, \\
& \partial_{t} u^{n+1}+u^{n} \cdot \nabla u^{n+1}+\nabla p^{n+1}-\Delta u^{n+1}=-\int_{\mathbb{R}^{3}} F_{d}\left[u^{n}\right]\left(f_{1}^{n+1}+f_{2}^{n+1}\right) d \xi, \\
& \quad x \in \Omega, t>0, \quad \nabla \cdot u^{n+1}=0, \quad x \in \Omega, t>0,
\end{aligned}
$$

with initial data and first iterate:

$$
\begin{aligned}
& \left(f_{10}^{n}(x, \xi), f_{20}^{n}(x, \xi), u_{0}^{n}(x)\right)=\left(f_{10}(x, \xi), f_{20}(x, \xi), u_{0}(x)\right), \\
& (x, \xi) \in \Omega \times \mathbb{R}^{3}, \quad n \geq 1,
\end{aligned}
$$

and

$$
\begin{aligned}
& \left(f_{1}^{0}(x, \xi, t), f_{2}^{0}(x, \xi, t), u^{0}(x, t)\right)=\left(f_{10}(x, \xi), f_{20}(x, \xi), u_{0}(x)\right), \\
& \quad(x, \xi, t) \in \Omega \times \mathbb{R}^{3} \times[0, T) .
\end{aligned}
$$

Then the following proposition is an immediate consequence of Theorem 2.1.

Proposition 3.1. Suppose that the initial data $\left(f_{10}, f_{20}, u\right)$ satisfies (2.2). Then there exists a unique solution $\left(f_{1}^{n}, f_{2}^{n}, u^{n}\right)$ to the system (3.1)-(3.3) 
such that

$$
\begin{gathered}
\text { (i) }\left\|f_{1}^{n}\right\|_{L^{\infty}\left(0, T ; W^{1, \infty}\right)},\left\|f_{2}^{n}\right\|_{L^{\infty}\left(0, T ; W^{1, \infty}\right)} \leq \varepsilon^{\beta}, \\
(\text { ii })\left\|u^{n}\right\|_{\mathcal{C}\left([0, T] ; H^{2}\right)}+\left\|u^{n}\right\|_{L^{2}\left(0, T ; H^{3}\right)} \leq \varepsilon^{\alpha} \text { and } \\
\left\|u_{t}^{n}\right\|_{\mathcal{C}\left([0, T] ; L^{2}\right)}+\left\|u_{t}^{n}\right\|_{L^{2}\left(0, T ; H^{1}\right)} \leq \varepsilon^{\alpha^{-}}
\end{gathered}
$$

for all $n \geq 1$.

Proof. Since the smallness and regularity condition on the initial data $\left(f_{10}\right.$, $\left.f_{20}, u_{0}\right)$ are assumed, our iteration scheme is well-defined by Theorem 2.1.

We now provide the strong convergence of the approximated solutions $\left(f_{1}^{n}, f_{2}^{n}, u^{n}\right)_{n \geq 1}$.

Lemma 3.1. Let $\left(f_{1}^{n}, f_{2}^{n}, u^{n}\right)$ be the solution to the system (3.1)-(3.3) obtained from Proposition (3.1). Then the approximate solutions $\left(f_{1}^{n}, f_{2}^{n}, u^{n}\right)$ is Cauchy in $L^{\infty}\left(\Omega \times \mathbb{R}^{3} \times(0, T)\right) \times L^{\infty}\left(\Omega \times \mathbb{R}^{3} \times(0, T)\right) \times L^{\infty}\left(0, T ; H^{1}(\Omega)\right)$.

Proof. These estimates are quite similar to the ones in the proof of Theorem 2.1. We postpone its proof to Appendix A.

Proof of Theorem 1.1. $\diamond$ Existence.- From Lemma 3.1, we obtain that $\left(f_{1}^{n}\right.$, $\left.f_{2}^{n}\right)_{n \geq 1}$ and $\left(u^{n}\right)_{n \geq 1}$ are Cauchy sequences in $L^{\infty}\left(\Omega \times \mathbb{R}^{3} \times(0, T)\right)$ and $L^{\infty}\left(0, T ; H^{1}(\Omega)\right) \cap L^{2}\left(0, T ; H^{2}(\Omega)\right)$, respectively. Then there exist the limit functions $\left(f_{1}, f_{2}, u\right)$ such that

$$
f_{1}^{n} \rightarrow f_{1}, f_{2}^{n} \rightarrow f_{2} \quad \text { in } L^{\infty}\left(\Omega \times \mathbb{R}^{3} \times(0, T)\right),
$$

and

$$
u^{n} \rightarrow u \quad \text { in } L^{\infty}\left(0, T ; H^{1}(\Omega)\right) \cap L^{2}\left(0, T ; H^{2}(\Omega)\right) .
$$

Clearly, $\left(f_{1}, f_{2}, u\right)$ is a weak solution to the system (1.1)-(1.4). Thus in order to complete the proof of existence, it remains to show the limit functions $\left(f_{1}, f_{2}, u\right)$ are actually in $L^{\infty}\left(0, T ; W^{1, \infty}\left(\Omega \times \mathbb{R}^{3}\right)\right) \times L^{\infty}\left(0, T ; W^{1, \infty}(\Omega \times\right.$ $\left.\left.\mathbb{R}^{3}\right)\right) \times \mathcal{C}\left([0, T] ; H^{2}(\Omega)\right)$. We briefly give our strategy for this proof.

- $\left(f_{1}, f_{2}, u\right) \in L^{\infty}\left(0, T ; W^{1, \infty}\left(\Omega \times \mathbb{R}^{3}\right)\right) \times L^{\infty}\left(0, T ; W^{1, \infty}\left(\Omega \times \mathbb{R}^{3}\right)\right) \times$ $L^{\infty}\left(0, T ; H^{2}(\Omega)\right)$ : We first notice from the estimates of uniform bounds in Proposition 3.1 that for each $t \in[0, T]$ there exists a convergent subsequence $\left(f_{1}^{n_{k}}, f_{2}^{n_{k}}, u^{n_{k}}\right)$ such that

$$
\left(f_{1}^{n_{k}}(t), f_{2}^{n_{k}}(t), u^{n_{k}}(t)\right) \rightarrow\left(\bar{f}_{1}(t), \bar{f}_{2}(t), \bar{u}(t)\right) \quad \text { as } \quad k \rightarrow \infty
$$


for some $\left(\bar{f}_{1}(t), \bar{f}_{2}(t), \bar{u}(t)\right) \in W^{1, \infty}\left(\Omega \times \mathbb{R}^{3}\right) \times W^{1, \infty}\left(\Omega \times \mathbb{R}^{3}\right) \times H^{2}(\Omega)$. On the other hand, the convergence-estimates in Lemma 3.1 yield that

$$
\begin{array}{r}
\left(f_{1}^{n_{k}}(t), f_{2}^{n_{k}}(t), u^{n_{k}}(t)\right) \rightarrow\left(f_{1}(t), f_{2}(t), u(t)\right) \\
\quad \text { in } L^{\infty}\left(\Omega \times \mathbb{R}^{3}\right) \times L^{\infty}\left(\Omega \times \mathbb{R}^{3}\right) \times H^{1}(\Omega),
\end{array}
$$

as $k \rightarrow \infty$. Hence we have

$$
\begin{aligned}
& \left(\bar{f}_{1}(t), \bar{f}_{2}(t), \bar{u}(t)\right) \equiv\left(f_{1}(t), f_{2}(t), u(t)\right) \\
& \quad \text { in } W^{1, \infty}\left(\Omega \times \mathbb{R}^{3}\right) \times W^{1, \infty}\left(\Omega \times \mathbb{R}^{3}\right) \times H^{2}(\Omega),
\end{aligned}
$$

for each $t \in[0, T]$.

- $\left(u, u_{t}\right) \in \mathcal{C}\left([0, T] ; H^{2}(\Omega)\right) \times \mathcal{C}\left([0, T] ; L^{2}(\Omega)\right)$ : From the previous step, we have the existence of $u \in L^{\infty}\left(0, T ; H^{2}(\Omega)\right) \cap L^{2}\left(0, T ; H^{3}(\Omega)\right)$. Then it follows from the momentum equations $(1.1)_{3}$ that $u_{t} \in$ $L^{\infty}\left(0, T ; L^{2}(\Omega)\right) \cap L^{2}\left(0, T ; H^{1}(\Omega)\right)$. We now apply the same arguments in Step E in the proof of Theorem 2.1 to have the desired regularity.

$\diamond$ Uniqueness.- Let $\left(f_{1}, f_{2}, u\right)$ and $\left(\bar{f}_{1}, \bar{f}_{2}, \bar{u}\right)$ be the two strong solutions obtained in the part of existence proof with the same initial data. We set the differences between two solutions:

$$
\Delta(t):=\left\|f_{1}-\bar{f}_{1}\right\|_{L^{\infty}}^{2}+\left\|f_{2}-\bar{f}_{2}\right\|_{L^{\infty}}^{2}+\|u-\bar{u}\|_{H^{1}}^{2} .
$$

Then it follows from the estimate in Appendix A that

$$
\Delta(t) \leq C \int_{0}^{t} \Delta(s) d s, \quad \Delta(0)=0 .
$$

This yields $\Delta(t)=0$ for all time $t \in[0, T]$, i.e.,

$$
f_{i} \equiv \bar{f}_{i} \quad \text { in } L^{\infty}\left(\Omega \times \mathbb{R}^{3} \times(0, T)\right) \quad \text { and } \quad u \equiv \bar{u} \quad \text { in } \mathcal{C}\left([0, T] ; H^{1}(\Omega)\right) .
$$

Hence we easily conclude

$$
f_{i} \equiv \bar{f}_{i} \quad \text { in } W^{1, \infty}\left(\Omega \times \mathbb{R}^{3} \times(0, T)\right) \quad \text { and } \quad u \equiv \bar{u} \quad \text { in } \mathcal{C}\left([0, T] ; H^{2}(\Omega)\right) .
$$

This completes the proof. 


\section{Large-time behavior of solutions}

In this section, we explore the large-time behavior of solutions. In particular, we consider the periodic spatial domain $\mathbb{T}^{3}$.

Lemma 4.1. Let $\left(f_{1}, f_{2}, u\right)$ be the classical solutions to the system (1.1) satisfying

(i) $\left\|f_{10}\right\|_{L^{1}},\left\|f_{20}\right\|_{L^{1}}<\infty$.

(ii) $\lim _{|\xi| \rightarrow \infty}|\xi|^{2}\left(f_{1}(x, \xi, t)+f_{2}(x, \xi, t)\right)=0, \quad(x, t) \in \mathbb{T}^{3} \times[0, T)$.

Then we have

$$
\begin{aligned}
& \text { (i) } \int_{\mathbb{T}^{3} \times \mathbb{R}^{3}} f_{i}(x, \xi, t) d x d \xi=\int_{\mathbb{T}^{3} \times \mathbb{R}^{3}} f_{i 0}(x, \xi) d x d \xi, \quad i=1,2 . \\
& \text { (ii) } \frac{d}{d t}\left(\int_{\mathbb{T}^{3} \times \mathbb{R}^{3}} \xi\left(f_{1}+f_{2}\right) d x d \xi+\int_{\mathbb{T}^{3}} u d x\right)=0 .
\end{aligned}
$$

Lemma 4.2. Let $\left(f_{1}, f_{2}, u\right)$ be the classical solutions to the system (1.1) satisfying

(i) $\left\|f_{10}\right\|_{L^{1}}=\left\|f_{20}\right\|_{L^{1}}=1$.

(ii) $\lim _{|\xi| \rightarrow \infty}|\xi|^{2}\left(f_{1}(x, \xi, t)+f_{2}(x, \xi, t)\right)=0, \quad(x, t) \in \mathbb{T}^{3} \times[0, T)$.

Then we have

(i) $\quad \frac{1}{2} \frac{d}{d t} \int_{\mathbb{T}^{3} \times \mathbb{R}^{3}}\left|\xi-\xi_{c}^{i}\right| f_{i} d x d \xi$

(ii) $\frac{1}{2} \frac{d}{d t} \int_{T^{3}}\left|u-u_{c}\right|^{2} d x$

$$
\leq-\psi_{i}^{m} \int_{\mathbb{T}^{3} \times \mathbb{R}^{3}}\left|\xi-\xi_{c}^{i}\right| f_{i} d x d \xi+\int_{\mathbb{T}^{3} \times \mathbb{R}^{3}}\left(\xi-\xi_{c}^{i}\right) \cdot(u-\xi) f_{i} d x d \xi,
$$

$$
=-\int_{\mathbb{T}^{3}}|\nabla u|^{2} d x+\int_{\mathbb{T}^{3} \times \mathbb{R}^{3}}\left(u_{c}-u\right) \cdot(u-\xi)\left(f_{1}+f_{2}\right) d x d \xi,
$$

(iii) $\frac{1}{4} \frac{d}{d t}\left|u_{c}-\xi_{c}^{1}-\xi_{c}^{2}\right|^{2}=-\int_{\mathbb{T}^{3} \times \mathbb{R}^{3}}\left(u_{c}-\xi_{c}^{1}-\xi_{c}^{2}\right) \cdot(u-\xi)\left(f_{1}+f_{2}\right) d x d \xi$,

where $\psi_{i}^{m}$ is a nonnegative constant defined by

$$
\psi_{i}^{m}:=\inf _{(x, y) \in \mathbb{T}^{3} \times \mathbb{T}^{3}} \psi_{i}(x, y)
$$


Proof. Straightforward computations yield the results, so we omit here.

Proof of Theorem 1.2. Summing up all of the terms in Lemma 4.2, we obtain

$$
\begin{aligned}
& \frac{1}{2} \frac{d}{d t}\left(\int_{\mathbb{T}^{3} \times \mathbb{R}^{3}}\left|\xi-\xi_{c}^{1}\right|^{2} f_{1}+\left|\xi-\xi_{c}^{2}\right|^{2} f_{2} d x d \xi\right. \\
& \left.\quad+\int_{\mathbb{T}^{3}}\left|u-u_{c}\right|^{2} d x+\frac{1}{2}\left|u_{c}-\xi_{c}^{1}-\xi_{c}^{2}\right|^{2}\right) \\
& \leq-\psi_{1}^{m} \int_{\mathbb{T}^{3} \times \mathbb{R}^{3}}\left|\xi-\xi_{c}^{1}\right| f_{1} d x d \xi-\psi_{2}^{m} \int_{\mathbb{T}^{3} \times \mathbb{R}^{3}}\left|\xi-\xi_{c}^{2}\right| f_{i} d x d \xi \\
& \quad-\int_{\mathbb{T}^{3}}|\nabla u|^{2} d x-\int_{\mathbb{T}^{3} \times \mathbb{R}^{3}}|u-\xi|^{2}\left(f_{1}+f_{2}\right) d x d \xi .
\end{aligned}
$$

On the other hand, we find

$$
\begin{aligned}
- & \int_{\mathbb{T}^{3} \times \mathbb{R}^{3}}|u-\xi|^{2}\left(f_{1}+f_{2}\right) d x d \xi \\
\leq & \int_{\mathbb{T}^{3}}\left(\rho_{f_{1}}+\rho_{f_{2}}\right)\left|u-u_{c}\right|^{2} d x \\
& -\frac{1}{2}\left|u_{c}-\xi_{c}^{1}-\xi_{c}^{2}\right|^{2}-\frac{1}{2} \int_{\mathbb{T}^{3} \times \mathbb{R}^{3}}\left|\xi-\xi_{c}^{1}\right| f_{1}+\left|\xi-\xi_{c}^{2}\right| f_{2} d x d \xi \\
\leq & C \| \rho_{f_{1}}+\left.\rho_{f_{2}}\right|_{L^{\frac{3}{2}}} \int_{\mathbb{T}^{3}}|\nabla u|^{2} d x \\
& -\frac{1}{2}\left|u_{c}-\xi_{c}^{1}-\xi_{c}^{2}\right|^{2}-\frac{1}{2} \int_{\mathbb{T}^{3} \times \mathbb{R}^{3}}\left|\xi-\xi_{c}^{1}\right| f_{1}+\left|\xi-\xi_{c}^{2}\right| f_{2} d x d \xi,
\end{aligned}
$$

where we used

$$
\begin{aligned}
\left\|\sqrt{\rho_{f_{1}}+\rho_{f_{2}}}\left(u-u_{c}\right)\right\|_{L^{2}} & \leq\left\|\sqrt{\rho_{f_{1}}+\rho_{f_{2}}}\right\|_{L^{3}}\left\|u-u_{c}\right\|_{L^{6}} \\
& \leq C\left\|\rho_{f_{1}}+\rho_{f_{2}}\right\|_{L^{\frac{3}{2}}}^{\frac{1}{2}}\|u\|_{H^{1}} \\
& \leq C\left\|\rho_{f_{1}}+\rho_{f_{2}}\right\|_{L^{\frac{3}{2}}}^{\frac{1}{2}}\|\nabla u\|_{L^{2} .}
\end{aligned}
$$

This yields

$$
\begin{aligned}
\frac{1}{2} \frac{d}{d t} & \left(\int_{\mathbb{T}^{3} \times \mathbb{R}^{3}}\left|\xi-\xi_{c}^{1}\right|^{2} f_{1}+\left|\xi-\xi_{c}^{2}\right|^{2} f_{2} d x d \xi\right. \\
& \left.+\int_{\mathbb{T}^{3}}\left|u-u_{c}\right|^{2} d x+\frac{1}{2}\left|u_{c}-\xi_{c}^{1}-\xi_{c}^{2}\right|^{2}\right)
\end{aligned}
$$




$$
\begin{aligned}
\leq & -\left(\psi_{1}^{m}+\frac{1}{2}\right) \int_{\mathbb{T}^{3} \times \mathbb{R}^{3}}\left|\xi-\xi_{c}^{1}\right| f_{1} d x d \xi-\left(\psi_{2}^{m}+\frac{1}{2}\right) \int_{\mathbb{T}^{3} \times \mathbb{R}^{3}}\left|\xi-\xi_{c}^{2}\right| f_{i} d x d \xi \\
& -C\left(1-C\left\|\rho_{f_{1}}+\rho_{f_{2}}\right\|_{L^{\frac{3}{2}}}\right) \int_{\mathbb{T}^{3}}\left|u-u_{c}\right|^{2} d x-\frac{1}{2}\left|u_{c}-\xi_{c}^{1}-\xi_{c}^{2}\right|^{2} .
\end{aligned}
$$

Hence if $\left\|\rho_{f_{1}}+\rho_{f_{2}}\right\|_{L^{\frac{3}{2}}}$ is small enough, then we conclude the desired result.

Remark 4.1. Even if $\psi_{i}^{m}=0$, we still have the exponential alignment between the particles and fluid. The reason is that the drag forces in particle and fluid equations play a role as the alignment force between particles and fluid. On the other hand, we notice that if $\psi_{i}^{m}>0$, then we can expect that the decay exponent for the alignment becomes large, and it makes them to align faster.

\section{Conclusion}

In this paper, we presented a new particle-fluid equations which describes the interactions between the two-species flocking particles and incompressible viscous fluid. For this model, we proved the global existence of the unique strong solution for sufficiently small and regular initial data. We also established the large-time behavior of the classical solutions under suitable assumptions. It would be an interesting problem if we consider other interaction forces between two-species particle, such as repulsive, attractive, self-propulsion and friction forces. We will leave these interesting issues to our future work.

\section{Acknowledgments}

Y.-P. Choi was supported by Basic Science Research Program through the National Research Foundation of Korea (NRF) funded by the Ministry of Education, Science and Technology (2012R1A6A3A03039496). B. Kwon was supported by Basic Science Research Program through the National Research Foundation of Korea (NRF) funded by the Ministry of Education, Science and Technology (NRF-2012R1A1A1015116). This work was also supported by the year of 2012 Research Fund of the Ulsan National Institute of Science and Technology (UNIST).

\section{Appendix A. Proof of Lemma 3.1}

In this part, we provide the detailed proof of Lemma 3.1.

- Step A.- Estimate of the bound of $\left\|f_{i}^{n+1}(t)-f_{i}^{n}(t)\right\|_{L^{\infty}}$ : Using the similar notations in the proof of Lemma 2.3, we find 


$$
\begin{aligned}
\mathcal{N}_{i}\left(f_{i}^{n+1}-f_{i}^{n}\right)= & -\nabla_{\xi} \cdot\left(F_{a}^{i}\left[f_{i}^{n+1}\right]+F_{d}\left[u^{n}\right]\right)\left(f_{i}^{n+1}-f_{i}^{n}\right) \\
& +\nabla_{\xi} \cdot\left(F_{a}^{i}\left[f_{i}^{n}\right]-F_{a}^{i}\left[f_{i}^{n+1}\right]\right) f_{i}^{n} \\
& +\left(F_{a}^{i}\left[f_{i}^{n}\right]-F_{a}^{i}\left[f_{i}^{n+1}\right]\right) \cdot \nabla_{\xi} f_{i}^{n}-\left(u^{n}-u^{n-1}\right) \cdot \nabla_{\xi} f_{i}^{n} \\
= & : \sum_{j=1}^{4} I_{j}^{i} .
\end{aligned}
$$

Here $I_{j}^{i}, j=1, \cdots, 4$ are estimated as follows.

$$
\begin{aligned}
I_{1}^{i} & =3\left(\int_{\Omega \times \mathbb{R}^{3}} \psi_{i}(x, y) f_{i}^{n+1} d y d \xi_{*}\right)\left(f_{i}^{n+1}-f_{i}^{n}\right) \\
& \leq 3\left(\left\|\psi_{i}\right\|_{L^{\infty}} M_{i 0}+1\right)\left\|f_{i}^{n+1}-f_{i}^{n}\right\|_{L^{\infty}}, \\
I_{2}^{i} & =3\left(\int_{\Omega \times \mathbb{R}^{3}} \psi_{i}(x, y)\left(f_{i}^{n+1}-f_{i}^{n}\right) d y d \xi_{*}\right) f_{i}^{n} \\
& \leq 3 \pi\left(R_{\xi}^{i, \infty}\right) \pi\left(R_{x}^{i, \infty}\right)\left\|f_{i}^{n+1}-f_{i}^{n}\right\|_{L^{\infty}}\left\|\psi_{i}\right\|_{L^{\infty}}\left\|f_{i}^{n}\right\|_{L^{\infty}} \leq C\left\|f_{i}^{n+1}-f_{i}^{n}\right\|_{L^{\infty}}, \\
I_{3}^{i} & =\left(\int_{\Omega \times \mathbb{R}^{3}} \psi_{i}(x, y)\left(\xi-\xi_{*}\right)\left(f_{i}^{n+1}-f_{i}^{n}\right) d y d \xi_{*}\right) \cdot \nabla_{\xi} f_{i}^{n} \\
& \leq 2\left\|\psi_{i}\right\|_{L^{\infty}} R_{\xi}^{i, \infty} \pi\left(R_{\xi}^{i, \infty}\right) \pi\left(R_{x}^{i, \infty}\right)\left\|f_{i}^{n+1}-f_{i}^{n}\right\|_{L^{\infty}}\left|\nabla_{\xi} f_{i}^{n}\right| \\
& \leq C\left\|f_{i}^{n+1}-f_{i}^{n}\right\|_{L^{\infty}}, \\
I_{4}^{i} & \leq\left\|u^{n}-u^{n-1}\right\|_{L^{\infty}}\left|\nabla_{\xi} f_{i}^{n}\right| \leq C\left\|u^{n}-u^{n-1}\right\|_{L^{\infty}} .
\end{aligned}
$$

This yields

$\left\|f_{i}^{n+1}(t)-f_{i}^{n}(t)\right\|_{L^{\infty}} \leq C\left\|u^{n}(t)-u^{n-1}(t)\right\|_{L^{\infty}}+C \int_{0}^{t}\left\|f_{i}^{n+1}(s)-f_{i}^{n}(s)\right\|_{L^{\infty}} d s$,

in turn, we have

$$
\left\|f_{i}^{n+1}(t)-f_{i}^{n}(t)\right\|_{L^{\infty}} \leq C \int_{0}^{t}\left\|u^{n}(s)-u^{n-1}(s)\right\|_{H^{2}} d s \quad \text { for all } \quad t \in[0, T] .
$$

- Step B.- Estimate of the bound of $\left\|u^{n+1}-u^{n}\right\|_{H^{1}}^{2}$ : It follows from (3.1) that

$$
\begin{gathered}
\partial_{t}\left(u^{n+1}-u^{n}\right)-\Delta\left(u^{n+1}-u^{n}\right)+\nabla\left(p^{n+1}-p^{n}\right) \\
=-u^{n} \cdot \nabla\left(u^{n+1}-u^{n}\right)-\left(u^{n}-u^{n-1}\right) \cdot \nabla u^{n} \\
-\int_{\mathbb{R}^{3}}\left(u^{n}-u^{n-1}\right)\left(f_{1}^{n+1}+f_{2}^{n+1}\right) d \xi
\end{gathered}
$$




$$
\begin{gathered}
-\int_{\mathbb{R}^{3}} u^{n-1}\left(f_{1}^{n+1}-f_{1}^{n}+f_{2}^{n+1}-f_{2}^{n}\right) d \xi \\
+\int_{\mathbb{R}^{3}} \xi\left(f_{1}^{n+1}-f_{1}^{n}+f_{2}^{n+1}-f_{2}^{n}\right) d \xi, \\
\nabla \cdot\left(u^{n+1}-u^{n}\right)=0, \quad t>0, \quad x \in \Omega .
\end{gathered}
$$

$\diamond$ Substep B1.- Zeroth-order estimate: Similarly as in Step A for the proof of Theorem 2.1, we find

$$
\begin{aligned}
\frac{1}{2} \frac{d}{d t} \| & u^{n+1}-u^{n}\left\|_{L^{2}}^{2}+\right\| \nabla\left(u^{n+1}-u^{n}\right) \|_{L^{2}}^{2} \\
\leq & C\left\|\nabla u^{n}\right\|_{L^{2}}\left\|u^{n}-u^{n-1}\right\|_{L^{3}}\left\|u^{n+1}-u^{n}\right\|_{L^{6}} \\
& +C\left(\left\|f_{1}^{n+1}\right\|_{L^{\infty}}+\left\|f_{2}^{n+1}\right\|_{L^{\infty}}\right)\left\|u^{n}-u^{n-1}\right\|_{L^{2}}\left\|u^{n+1}-u^{n}\right\|_{L^{2}} \\
& +C\left(\left\|f_{1}^{n+1}-f_{1}^{n}\right\|_{L^{\infty}}+\left\|f_{2}^{n+1}-f_{2}^{n}\right\|_{L^{\infty}}\right)\left(\left\|u^{n-1}\right\|_{L^{2}}+1\right)\left\|u^{n+1}-u^{n}\right\|_{L^{2}} \\
\leq & C \varepsilon^{\alpha}\left\|u^{n}-u^{n-1}\right\|_{H^{1}}\left\|\nabla\left(u^{n+1}-u^{n}\right)\right\|_{L^{2}} \\
& +C \varepsilon^{\beta}\left\|u^{n}-u^{n-1}\right\|_{L^{2}}\left\|u^{n+1}-u^{n}\right\|_{L^{2}} \\
& +C\left(1+\varepsilon^{\alpha}\right)\left(\left\|f_{1}^{n+1}-f_{1}^{n}\right\|_{L^{\infty}}+\left\|f_{2}^{n+1}-f_{2}^{n}\right\|_{L^{\infty}}\right)\left\|u^{n+1}-u^{n}\right\|_{L^{2}} \\
\leq & \frac{1}{2}\left\|\nabla\left(u^{n+1}-u^{n}\right)\right\|_{L^{2}}^{2}+\frac{1}{2}\left\|u^{n+1}-u^{n}\right\|_{L^{2}}^{2}+C\left\|^{n}-u^{n-1}\right\|_{H^{1}}^{2} \\
& +C\left\|f_{1}^{n+1}-f_{1}^{n}\right\|_{L^{\infty}}^{2}+C\left\|f_{2}^{n+1}-f_{2}^{n}\right\|_{L^{\infty}}^{2} .
\end{aligned}
$$

This yields

$$
\begin{aligned}
& \frac{d}{d t}\left\|u^{n+1}-u^{n}\right\|_{L^{2}}^{2}+\left\|\nabla\left(u^{n+1}-u^{n}\right)\right\|_{L^{2}}^{2} \\
& \leq C\left\|u^{n+1}-u^{n}\right\|_{L^{2}}^{2}+C\left\|u^{n}-u^{n-1}\right\|_{H^{1}}^{2} \\
& \quad+C\left\|f_{1}^{n+1}-f_{1}^{n}\right\|_{L^{\infty}}^{2}+C\left\|f_{2}^{n+1}-f_{2}^{n}\right\|_{L^{\infty}}^{2} .
\end{aligned}
$$

$\diamond$ Substep B2.- First-order estimate: We again use a similar argument in Step B for the proof of Theorem 2.1 to deduce

$$
\begin{aligned}
\frac{1}{2} \frac{d}{d t} \| & \nabla\left(u^{n+1}-u^{n}\right)\left\|_{L^{2}}^{2}+\right\| \nabla^{2}\left(u^{n+1}-u^{n}\right) \|_{L^{2}}^{2} \\
= & -\int_{\Omega} \nabla\left(u^{n+1}-u^{n}\right): \nabla\left(u^{n} \cdot \nabla\left(u^{n+1}-u^{n}\right)\right) d x \\
& -\int_{\Omega} \nabla\left(u^{n+1}-u^{n}\right): \nabla\left(\left(u^{n}-u^{n-1}\right) \cdot \nabla u^{n}\right) d x \\
& -\int_{\Omega \times \mathbb{R}^{3}} \nabla\left(u^{n+1}-u^{n}\right): \nabla\left(\left(u^{n}-u^{n-1}\right)\left(f_{1}^{n+1}+f_{2}^{n+1}\right)\right) d \xi d x
\end{aligned}
$$




$$
\begin{aligned}
& -\int_{\Omega \times \mathbb{R}^{3}} \nabla\left(u^{n+1}-u^{n}\right): \nabla\left(u^{n-1}\left(f_{1}^{n+1}-f_{1}^{n}+f_{2}^{n+1}-f_{2}^{n}\right)\right) d \xi d x \\
& +\int_{\Omega \times \mathbb{R}^{3}} \nabla\left(u^{n+1}-u^{n}\right): \xi \otimes \nabla\left(f_{1}^{n+1}-f_{1}^{n}+f_{2}^{n+1}-f_{2}^{n}\right) d \xi d x \\
= & \sum_{j=1}^{5} J_{j} .
\end{aligned}
$$

where $J_{j}, j=1, \cdots, 5$ are estimated by

$$
\begin{aligned}
J_{1} \leq & \left\|\nabla\left(u^{n+1}-u^{n}\right)\right\|_{L^{3}}\left\|\nabla u^{n}\right\|_{L^{2}}\left\|\nabla\left(u^{n+1}-u^{n}\right)\right\|_{L^{6}} \\
\leq & C\left\|u^{n}\right\|_{H^{1}}\left\|\nabla\left(u^{n+1}-u^{n}\right)\right\|_{H^{1}}^{2} \leq C \varepsilon^{\alpha}\left\|\nabla\left(u^{n+1}-u^{n}\right)\right\|_{H^{1}}^{2}, \\
J_{2} \leq & \left\|\nabla\left(u^{n+1}-u^{n}\right)\right\|_{L^{3}}\left(\left\|\nabla u^{n}\right\|_{L^{6}}\left\|\nabla\left(u^{n}-u^{n-1}\right)\right\|_{L^{2}}\right. \\
& \left.+\left\|\nabla^{2} u^{n}\right\|_{L^{2}}\left\|u^{n}-u^{n-1}\right\|_{L^{6}}\right) \\
\leq & C \varepsilon^{\alpha}\left\|u^{n}-u^{n-1}\right\|_{H^{1}}\left\|\nabla\left(u^{n+1}-u^{n}\right)\right\|_{H^{1}}, \\
J_{3} \leq & C\left\|\nabla\left(u^{n+1}-u^{n}\right)\right\|_{L^{2}}\left(\left\|f_{1}^{n+1}\right\|_{L^{\infty}}+\left\|f_{2}^{n+1}\right\|_{L^{\infty}}\right)\left\|\nabla\left(u^{n}-u^{n-1}\right)\right\|_{L^{2}} \\
& +C\left\|\nabla\left(u^{n+1}-u^{n}\right)\right\|_{L^{2}}\left(\left\|\nabla f_{1}^{n+1}\right\|_{L^{\infty}}+\left\|\nabla f_{2}^{n+1}\right\|_{L^{\infty}}\right)\left\|u^{n}-u^{n-1}\right\|_{L^{2}} \\
\leq & C \varepsilon^{\beta}\left\|u^{n}-u^{n-1}\right\|_{H^{1}}\left\|\nabla\left(u^{n+1}-u^{n}\right)\right\|_{L^{2}}, \\
J_{4} \leq & \int_{\Omega \times \mathbb{R}^{3}}\left|\nabla^{2}\left(u^{n+1}-u^{n}\right)\left\|u^{n-1}\right\| f_{1}^{n+1}-f_{1}^{n}+f_{2}^{n+1}-f_{2}^{n}\right| d x d \xi \\
\leq & C\left(\left\|f_{1}^{n+1}-f_{1}^{n}\right\|_{L^{\infty}}+\left\|f_{2}^{n+1}-f_{2}^{n}\right\|_{L^{\infty}}\right)\left\|\nabla^{2}\left(u^{n+1}-u^{n}\right)\right\|_{L^{2}}\left\|u^{n-1}\right\|_{L^{2}} \\
\leq & C \varepsilon^{\alpha}\left(\left\|f_{1}^{n+1}-f_{1}^{n}\right\|_{L^{\infty}}+\left\|f_{2}^{n+1}-f_{2}^{n}\right\|_{L^{\infty}}\right)\left\|\nabla\left(u^{n+1}-u^{n}\right)\right\|_{H^{1}}, \\
J_{5} \leq & \int_{\Omega \times \mathbb{R}^{3}}\left|\nabla^{2}\left(u^{n+1}-u^{n}\right)\|\xi\| f_{1}^{n+1}-f_{1}^{n}+f_{2}^{n+1}-f_{2}^{n}\right| d \xi d x \\
\leq & C\left(\left\|f_{1}^{n+1}-f_{1}^{n}\right\|_{L^{\infty}}+\left\|f_{2}^{n+1}-f_{2}^{n}\right\|_{L^{\infty}}\right)\left\|\nabla\left(u^{n+1}-u^{n}\right)\right\|_{H^{1}} .
\end{aligned}
$$

Thus we obtain

$$
\begin{aligned}
\frac{1}{2} \frac{d}{d t} \| & \nabla\left(u^{n+1}-u^{n}\right)\left\|_{L^{2}}^{2}+\right\| \nabla^{2}\left(u^{n+1}-u^{n}\right) \|_{L^{2}}^{2} \\
\leq & C\left\|\nabla\left(u^{n+1}-u^{n}\right)\right\|_{L^{2}}^{2}+\frac{1}{2}\left\|\nabla^{2}\left(u^{n+1}-u^{n}\right)\right\|_{L^{2}}^{2} \\
& +C\left(\left\|u^{n}-u^{n-1}\right\|_{H^{1}}^{2}+\left\|f_{1}^{n+1}-f_{1}^{n}\right\|_{L^{\infty}}^{2}+\left\|f_{2}^{n+1}-f_{2}^{n}\right\|_{L^{\infty}}^{2}\right),
\end{aligned}
$$

and

$$
\frac{d}{d t}\left\|\nabla\left(u^{n+1}-u^{n}\right)\right\|_{L^{2}}^{2}+\left\|\nabla^{2}\left(u^{n+1}-u^{n}\right)\right\|_{L^{2}}^{2}
$$




$$
\begin{aligned}
\leq & C\left(\left\|\nabla\left(u^{n+1}-u^{n}\right)\right\|_{L^{2}}^{2}+\left\|u^{n}-u^{n-1}\right\|_{H^{1}}^{2}\right. \\
& \left.+\left\|f_{1}^{n+1}-f_{1}^{n}\right\|_{L^{\infty}}^{2}+\left\|f_{2}^{n+1}-f_{2}^{n}\right\|_{L^{\infty}}^{2}\right) .
\end{aligned}
$$

We finally combine (A.1), (A.2) and (A.3) to find

$$
\begin{aligned}
& \frac{d}{d t}\left\|u^{n+1}-u^{n}\right\|_{H^{1}}^{2}+\left\|\nabla\left(u^{n+1}-u^{n}\right)\right\|_{H^{1}}^{2} \\
& \leq C\left(\left\|u^{n+1}-u^{n}\right\|_{H^{1}}^{2}+\left\|u^{n}-u^{n-1}\right\|_{H^{1}}^{2}+\left\|f_{1}^{n+1}-f_{1}^{n}\right\|_{L^{\infty}}^{2}\right. \\
& \left.\quad+\left\|f_{2}^{n+1}-f_{2}^{n}\right\|_{L^{\infty}}^{2}\right) \\
& \leq C\left(\left\|u^{n+1}-u^{n}\right\|_{H^{1}}^{2}+\left\|u^{n}-u^{n-1}\right\|_{H^{1}}^{2}+\int_{0}^{t}\left\|u^{n}-u^{n-1}\right\|_{H^{2}}^{2} d s\right) .
\end{aligned}
$$

This implies

$$
\begin{aligned}
& \left\|u^{n+1}-u^{n}\right\|_{H^{1}}^{2}+\int_{0}^{t}\left\|\nabla\left(u^{n+1}-u^{n}\right)\right\|_{H^{1}}^{2} d s \\
& \leq C\left(\int_{0}^{t}\left\|u^{n+1}-u^{n}\right\|_{H^{1}}^{2} d s+\int_{0}^{t}\left\|u^{n}-u^{n-1}\right\|_{H^{1}}^{2} d s\right. \\
& \left.\quad+\int_{0}^{t} \int_{0}^{s}\left\|\nabla\left(u^{n}-u^{n-1}\right)\right\|_{H^{1}}^{2} d \tau d s\right) .
\end{aligned}
$$

Applying the Gronwall's inequality for $\left\|u^{n+1}-u^{n}\right\|_{H^{1}}^{2}$, and using the iteration argument for the resulting inequality, we obtain

$\left\|u^{n+1}-u^{n}\right\|_{L^{\infty}\left(0, T ; H^{1}\right)}+\left\|\nabla\left(u^{n+1}-u^{n}\right)\right\|_{L^{2}\left(0, T ; H^{1}\right)} \leq \frac{(C(T))^{n+1}}{n !}$ for all $n \geq 0$.

This together with (A.1) also implies that

$$
\left\|f_{1}^{n+1}-f_{1}^{n}\right\|_{L^{\infty}}+\left\|f_{2}^{n+1}-f_{2}^{n}\right\|_{L^{\infty}} \leq \frac{(C(T))^{n+1}}{n !} \text { for all } n \geq 0 .
$$

Here $C(T)$ denotes the positive constant depending only on $T>0$. By (A.4) and (A.5), one can conclude that $\left\{u^{n}\right\}$ and $\left\{f_{i}^{n}\right\}$ are Cauchy sequences in the desired spaces. This completes the proof.

\section{References}

[1] C. Baranger, L. Boudin, P.-E. Jabin, and S. Mancini, A modeling of biospray for the upper airways, ESAIM Proc., 14, (2005), 41-47. MR2226800 
[2] H.-O. Bae, Y.-P. Choi, S.-Y. Ha, and M.-J. Kang, Time-asymptotic interaction of flocking particles and an incompressible viscous fluid, Nonlinearity, 24, (2012), 1155-1177. MR2904273

[3] H.-O. Bae, Y.-P. Choi, S.-Y. Ha, and M.-J. Kang, Global existence of strong solution for the Cucker-Smale-Navier-Stokes system, preprint.

[4] H.-O. Bae, Y.-P. Choi, S.-Y. Ha, and M.-J. Kang, Asymptotic flocking dynamics of Cucker-Smale particles immersed in compressible fluids, to appear in Discrete and Continuous Dynamical Systems-A.

[5] Birnir, An ODE model of the motion of pelagic fish, J. Stat. Phys., 128, (2007), 535-568. MR2331196

[6] L. Boudin, L. Desvillettes, C. Grandmont, and A. Moussa, Global existence of solution for the coupled Vlasov and Navier-Stokes equations, Differential and Integral Equations, 22, (2009), 1247-1271. MR2555647

[7] J. A. Carrillo, R. Duan, and A. Moussa, Global classical solutions close to the equilibrium to the Vlasov-Fokker-Planck-Euler system, Kinetic and Related Models, 4, (2011), 227-258. MR2765745

[8] J. A. Carrillo, T. K. Karper, and K. Trivisa, On the dynamics of a fluidparticle interaction model: The bubbling regime, Nonlinear Analysis: Theory, Methods \& Applications, 74(8), (2011), 2778-2801. MR2776527

[9] M. Chae, K. Kang, and J. Lee, Global existence of weak and classical solutions for the Navier-Stokes-Vlasov-Fokker-Planck equations, J. Differential Equations, 251, (2011), 2431-2465. MR2825335

[10] A. Chakraborti, Distributions of money in models of market economy, Int. J. Modern Phys. C, 13, (2002), 1315-1321.

[11] Y.-P. Choi and J. Lee, Global existence of weak and strong solutions to Cucker-Smale-Navier-Stokes equations in $\mathbb{R}^{2}$, preprint.

[12] C. Conca, E. Espejo, and K. Vilches, Remarks on the blowup and global existence for a two species chemotactic Keller-Segel system in R2, European J. Appl. Math., 22, (2011), 553-580. MR2853987

[13] P. Constantin, C. Fefferman, E. Titi, and A. Zarnescu, Regularity for coupled two dimensional nonlinear-Fokker-Planck and Navier-Stokes systems. Commun. Math. Phys., 270, (2007), 789-811. MR2276466

[14] I. D. Couzin, J. Krause, N. R. Franks, and S. A. Levin, Effective leadership and decision-making in animal groups on the move, Nature, 433, (2005), 513-516. 
[15] F. Cucker and S. Smale, Emergent behavior in flocks, IEEE Trans. Automat. Control, 52, (2007), 852-562. MR2324245

[16] P. Degond and S. Motsch, Continuum limit of self-driven particles with orientation interaction, Math. Models Methods Appl. Sci., 18, (2008), 1193-1215. MR2438213

[17] R. Duan, A. Lorz, and P. Markowich, Global solutions to the coupled chemotaxis-fluid equations, Comm. in PDE., 35, (2010), 1635-1673. MR2754058

[18] B. Düring, P. Markowich, J. F. Pietschmann, and M. T. Wolfram, Boltzmann and Fokker-Planck equations modelling opinion formation in the presence of strong leaders, Proceedings of the Royal Society A: Mathematical, Physical and Engineering Sciences, 485, (2009), 3687-3708. MR2552289

[19] C. Escudero, F. Maciá, and J. J. L. Velázquez, Two-species-coagulation approach to consensus by group level interactions, Physical Review E, 82, (2010), 016113. MR2736376

[20] E. E. Espejo, A. Stevens, and J. J. L. Velázquez, Simultaneous finite time blow-up in a two-species model for chemotaxis, Analysis, 29, (2009), 317-338. MR2568886

[21] M. Francesco and S. Fagiolo, Measure solutions for nonlocal interactions pdes with two speicies, Nonlinearity, 26, (2013), 2777-2808. MR3105514

[22] S.-Y. Ha, M.-J. Kang and B. Kwon, A hydrodynamic model for the interaction of Cucker-Smale particles and incompressible fluids, to appear in Math. Mod. and Meth. in Appl. Sci.

[23] D. Horstmann, Generalizing the Keller-Segel model: Lyapunov functionals, steady state analysis, and blow-up results for multi-species chemotaxis models in the presence of attraction and repulsion between compretitive interacting species, J. Nonlinear Sci., 21, (2011), 231-270. MR2788856

[24] A. Jadbabaie, J. Lin, and A. S. Morse, Coordination of groups of mobile autonomous agents using nearest neighbor rules, IEEE Trans. Automat. Control, 48, (2003), 988-1001. MR1986266

[25] A. Mackey, T. Kolokolnikov, and A. Bertozzi, Two-species particle aggregation and stability of co-dimension one solutions, preprint. MR3199785 
[26] A. Mellet and A. Vasseur, Global weak solutions for a Vlasov-FokkerPlanck/Navier-Stokes system of equations, Math. Models Methods Appl. Sci., 17, (2007), 1039-1063. MR2337430

[27] A. Mogilner, L. Edelstein-Keshet, L. Bent, and A. Spiros, Mutual interactions, potentials, and individual distance in a social aggregation, Journal of Mathematical Biology, 47, (2003), 353-389. MR2024502

[28] L. Perea, G. Gómez, and P. Elosegui, Extension of the Cucker-Smale control law to space flight formations, AIAA J. Guid. Control Dynam., $32,(2009), 527-537$.

[29] M. C. Rinaldo, M. Garavello, and M. Lécureux-Mercier, A class of nonlocal models for pedestrian traffic, Math. Models and Methods in Appl. Sci., 22, (2012), 1150023. MR2902155

[30] K. Sznajd-Weron, and J. Sznajd, Opinion evolution in closed community, Int. J. Mod. Phys. C, 11, (2000), 1157-1165.

[31] J. Tello and M. Winkler, Stabilization in a two-species chemotaxis system with a logistic source, Nonlinearity, 25, (2012), 1413-1425. MR2914146

Young-Pil Choi

Department of Mathematics

IMPERIAL COLLEGE LONDON

LONDON SW7 2AZ

UNITED KINGDOM

E-mail address: young-pil.choi@imperial.ac.uk

Bongsuk KWON

Department of Mathematical Sciences

Ulsan National Institute of Science and Technology

ULSAN 689-798

KOREA

E-mail address: bkwon@unist.ac.kr

Received August 30, 2013 